\title{
Chatbot de orientações acerca do aleitamento materno: desenvolvimento e validação
}

\author{
Andréa de Oliveira Ribeiro Cavalcanti ${ }^{1,2}$, Andréia Roque de Souza Cavalcanti ${ }^{1,2}$, \\ José William Araújo do Nascimento, ${ }^{1,2}$, Rafael Roque de Souza ${ }^{1,2}$, Geicianfran da \\ Silva Lima Roque ${ }^{1,2}$, Sérgio Ricardo de Melo Queiroz ${ }^{2}$, Isabel Cristina Ramos \\ Vieira Santos ${ }^{3}$
}

${ }^{1}$ Imune Research Lab, João Pessoa, PB - Brasil

${ }^{2}$ Centro de Informática, Universidade Federal de Pernambuco (Cin-UFPE) - Recife, PE - Brasil

${ }^{3}$ Faculdade de Enfermagem Nossa Senhora das Graças, Universidade de Pernambuco (UPE) - Recife, PE - Brasil

andreacavalcantil4@gmail.com, andreia@imunekids.com.br, \{jwan; rrs4; gslr; srmq\} @cin.ufpe.br, tutornadeyahoo.com.br

\begin{abstract}
The proposed study aims to develop a chatbot to provide personalized guidance on breastfeeding. This is methodological research; the chatbot content development was derived from references in the literature related to the theme of breastfeeding and the computerization was performed by computer science experts, using the IBM Watson Conversation platform. The chatbot content obtained an average validation of 0.98 (Content Validity Index), indicating excellent confidence among the evaluators, making the prototype ready to be tested among users (pregnant and lactating women).
\end{abstract}

Resumo. $O$ estudo proposto tem por objetivo desenvolver um chatbot com a finalidade de fornecer orientações personalizadas acerca do aleitamento materno. Trata-se de uma pesquisa metodológica, cujo desenvolvimento do conteúdo do chatbot foi decorrente de referências da literatura relacionadas ao tema aleitamento materno e a informatização foi realizada por especialistas da ciência da computação, através da plataforma IBM Watson Conversation. $O$ conteúdo do chatbot obteve uma validação média de 0,98 (Índice de Validade de Conteúdo), indicando excelente confiança entre os avaliadores, fazendo com que o protótipo esteja pronto a ser testado entre usuários (gestantes e lactantes).

\section{Introdução}

O Aleitamento Materno (AM) é a forma mais natural de abastecimento ou alimento ideal para o crescimento e desenvolvimento da criança e também tem enorme influência biológica e emocional na saúde [Schincaglia et al., 2015]. Nas lactantes ele é responsável por diminuir o fator de risco para o câncer de mama e de ovário, reduzir as chances de desenvolver o diabetes mellitus tipo 2, além de aumentar o intervalo entre os partos. Já para os lactentes o AM oferece proteção contra infecções respiratórias, diarréia, má oclusão dentária, melhora do desenvolvimento cognitivo e diminuição das chances de excesso de peso e diabetes no futuro [Sena et al., 2020]. 
A Organização Mundial da Saúde recomenda o aleitamento materno de forma exclusiva nos primeiros 6 meses de vida e de forma complementar à dieta da criança até seus 2 anos ou mais de idade [WHO, 2009]. Porém, apesar das evidências científicas que comprovam a superioridade do aleitamento materno sobre as demais formas de alimentação da criança, os índices de amamentação nos países em desenvolvimento, especialmente no Brasil, são muito inferiores ao recomendado [Boccolini et al., 2017]. Os grandes desafios relacionados à amamentação, são considerados os fatores socioeconômico-culturais, desinformação da população e profissionais da área da saúde que não abordam as vantagens e importância do aleitamento materno [Santos et al., 2019]. Percebe-se então, a necessidade de inserção de novos materiais para auxílio aos profissionais de saúde na promoção e manejo do aleitamento materno e nesse contexto, o uso das tecnologias tem se mostrado eficaz [Tang et al., 2019].

$\mathrm{Na}$ área de Saúde, os chatbots baseados em inteligência artificial, podem desempenhar um papel estratégico na personalização do atendimento ao usuário. $\mathrm{O}$ desenvolvimento de um sistema computacional que permita captar e transmitir informações úteis à prática do aleitamento materno pode beneficiar a saúde dos lactentes e lactantes, bem como auxiliar os profissionais de saúde [Almohanna et al., 2020]. Desta forma, este estudo objetivou desenvolver um chatbot que visa a promoção e a orientação de condutas acerca do aleitamento materno. Especificamente os objetivos são: estruturar a base teórica de construção do assistente inteligente para orientação acerca do aleitamento materno; informatizar a base teórica através da utilização de um programa de computador, criando um chatbot; validar o conteúdo do chatbot.

\section{Métodos}

O estudo proposto desenha-se sob os moldes de uma pesquisa metodológica de escopo quantitativo. As etapas seguidas foram:

1) Revisão narrativa da literatura: foram consultados artigos científicos, materiais do Ministério da Saúde do Brasil e Organização Mundial da Saúde [Silva et al. 2020; Vilar et al., 2020].

2) Desenvolvimento do chatbot: foi construído na plataforma IBM Watson Conversation, dado a sua capacidade de processamento de linguagem natural, geração e avaliação de hipóteses e aprendizagem baseada na evidência [IBM, 2019]. Assim, os mecanismos de inteligência artificial utilizados foram: aprendizagem de máquina, que usa os dados para detectar padrões e ajustar as ações da máquina, estruturados ou nãoestruturados, que são coletados, armazenados e interpretados por sistemas especializados.

Ainda nesta etapa de desenvolvimento do chatbot, foram elaborados os intents. Uma intent, ou intenção, é a finalidade em que o usuário deseja chegar ao ter enviado uma mensagem, por exemplo, se o usuário enviar "Bom dia" ao chatbot, este deve saber que a intenção do usuário ao ter enviado aquela mensagem, foi de "saudação", ou, "início de diálogo. Para a elaboração destes intents recorremos ao roteiro com o fluxo de diálogos desenvolvido para registrar as intenções do usuário no diálogo. Para alimentar os intents , foram adicionadas palavras-chaves e respostas pré-definidas que auxiliem o usuário na retirada de dúvidas acerca do aleitamento materno.

3) Validação de conteúdo do chatbot: Um painel de 08 especialistas (médicos, enfermeiros e psicólogos) em aleitamento materno avaliaram o conteúdo do chatbot. A 
concordância dos especialistas foi verificada de forma quantitativa por meio do Índice de Validade de Conteúdo por item (I-IVC), considerando-se um resultado maior ou igual a 0,80 como valor mínimo adequado [Polit and Beck, 2006]. Além disso, foi utilizado o Índice de Validade de Conteúdo por nível de escala e média S-IVC/Ave). O S-IVC/Ave deve ser igual ou maior que 0,90 como o padrão para este índice de congruência média [Polit and Beck, 2006].

Para avaliar a confiabilidade entre as avaliações foi calculado o Interrater Agreement (IRA), que oferece uma perspectiva única de confiabilidade, já que lida com a consistência das avaliações e a concordância com a similaridade dos níveis absolutos de classificação. Para interpretação deste índice foi utilizado o recomendado por O'Neill [2017], que considera valores acima de 0,91 como de forte concordância. A estatística Kappa foi utilizada como complemento importante ao IVC, sendo considerado as seguintes interpretações dos valores: $>0,74$ - excelente; 0,60 a 0,74 - bom; 0,40 a 0,59 regular; < 0,40 - insuficiente [Nichols et al., 2010].

Os domínios do chatbot se referem aos eixos temáticos presentes no assistente virtual, descritos na tabela 1, enquanto os itens dos domínios são seus Requisitos Funcionais (RFs). De acordo com Pressman [2016], os RFs são as funções que o sistema deve fornecer, ou seja, como deverá reagir às entradas do usuário.

Tabela 1: Domínios do chatbot

\begin{tabular}{cl}
\hline Domínios (n de itens - comandos) & \multicolumn{1}{c}{ Descrição } \\
\hline Domínio 1 (3 itens) & Telas iniciais do Assistente Inteligente \\
Domínio 2 (20 itens) & $\begin{array}{l}\text { Orientações específicas do Assistente Inteligente } \\
\text { conforme escolha do usuário: }\end{array}$ \\
& $\begin{array}{l}\text { Dor mamária; dificuldade na pega; baixo ou alto } \\
\text { fluxo mamário; fissura mamária; ingurgitamento } \\
\text { mamário; fraca sucção do bebê; demora na descida } \\
\text { do leite; mamilos planos ou invertidos; aleitamento } \\
\text { e covid-19; vantagens da amamentação; tempo e } \\
\text { intervalo das mamadas; posições para o } \\
\text { aleitamento. }\end{array}$ \\
\hline
\end{tabular}

Este estudo foi aprovado pelo Comitê de Ética em Pesquisa da Faculdade de Enfermagem da Universidade de Pernambuco, Brasil (Número do Parecer: 4.512.452) e obedeceu aos preceitos do Código de Ética da Associação Médica Mundial (Declaração de Helsinque).

\section{Resultados}

\subsection{Validação de conteúdo}

Os oito especialistas que participaram do painel de validação de conteúdo do chatbot tinham uma média de idade de 44 anos (desvio padrão: 15,85 ), são do sexo feminino $(62,5 \%)$ e oriundos da região Nordeste $(87,5 \%)$.

Como primeira avaliação, os especialistas analisaram os domínios do conteúdo do chatbot de forma geral; verificou-se que estes domínios alcançaram critérios de adequação, relevância e representatividade excelentes, todos com 100\% de concordância. 
Em seguida os especialistas analisaram cada item (comando) isoladamente do chatbot; os 23 itens tiveram valores de IVC acima de 0,91 (valor mínimo: 0,91; valor máximo: 1,00), indicando excelente relevância do conteúdo. A avaliação da validade de conteúdo como um todo, pelo método de S-IVC/Ave alcançou uma média de 0,98, demonstrando que o conteúdo presente no chatbot apresenta um elevado índice de confiança entre especialistas da área.

A concordância inter-avaliadores apresentou uma concordância muito forte (IRA: 1,00 ) à semelhança dos resultados do Fleiss Kappa, no qual se verifica que todos os itens obtiveram concordância excelente, indicando concordância substancial entre os avaliadores. O índice de Kappa médio foi de 1,00 ( $\mathrm{p}<0,05)$.

\subsection{Protótipo do chatbot}

O Chatbot de orientações sobre o aleitamento materno (BotAleitamento), foi desenvolvido na plataforma IBM Watson Conversation, estando disponível atualmente na versão com interface web. Abaixo são apresentadas as duas seções presentes no chatbot.

Dificuldades das mães durante amamentação: Um conjunto de intents permite o diálogo com o usuário a fim de orientar sobre condutas corretas para amenizar situações indesejáveis que podem ocorrer durante amamentação (mastite, ingurgitamento, fissuras no mamilo, dentre outros).

Resolução de dúvidas das mães durante a amamentação: A sequência é apresentada através de uma série de instruções (roteiros) e figuras correspondentes que abordam o processo do aleitamento materno (posição correta para amamentar, realização da ordenha, como armazenar e doar o leite, dentre outros). Salienta-se que os usuários do chatbot podem interagir usando botões predefinidos ou digitando suas respostas. Quando a intenção do usuário não é identificada (por ter digitado uma resposta não reconhecida), o chatbot diz que não entendeu e repõe a pergunta, de modo que isso possa ocorrer a qualquer momento durante a conversa. $O$ fluxo da conversa foi programado pensando no público alvo (gestantes e lactantes) e todo o processo é fácil de entender para que o chatbot possa ser usado por pessoas de qualquer nível educacional. 


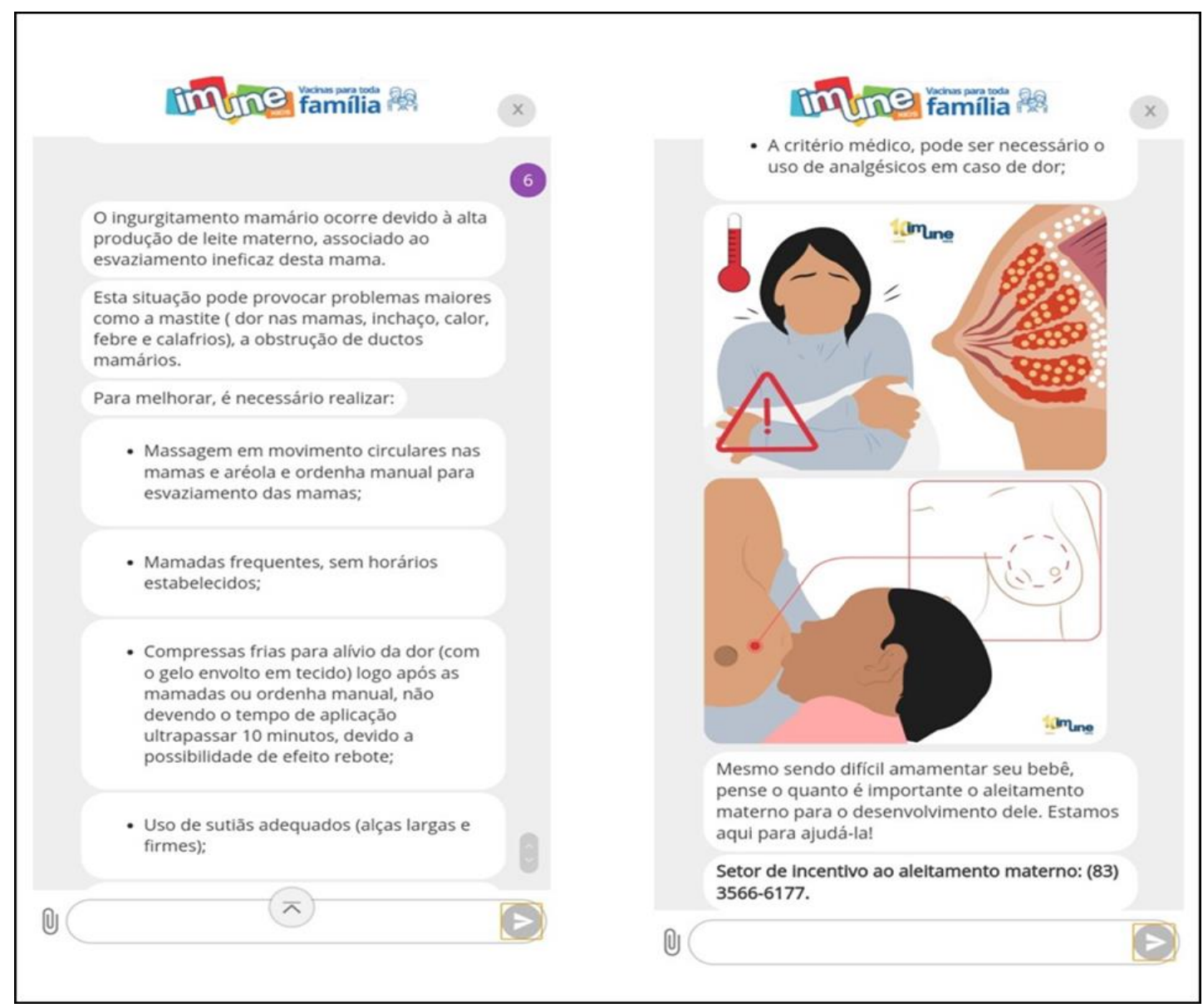

Figura 1: Interface web do chatbot: orientações acerca do ingurgitamento mamário

\section{Considerações finais}

Com base nos resultados, podemos concluir que o roteiro desenvolvido e incorporado no protótipo de chatbot "BotAleitamento" obteve um nível satisfatório de validação de conteúdo. O protótipo, atualmente, encontra-se pronto para os próximos testes, com usuários (gestantes, lactantes, puérperas e enfermeiros) para as devidas verificações de confiabilidade e usabilidade.

No futuro, pretendemos também desenvolver a interação por voz e vídeo entre os usuários e integrar o chatbot ao mensageiro Telegram, tendo em vista o crescimento na utilização no mercado de aplicativos de mensagens. Algumas das vantagens do Telegram são, além dos seus 200 milhões de usuários ativos mensais, seus recursos avançados de segurança e criptografia [Demartini, 2018].

\section{Referências}

Almohanna, A.A., Win, K.T. and Meedya, S. (2020) Effectiveness of Internet-Based Electronic Technology Interventions on Breastfeeding Outcomes: Systematic Review. J Med Internet Res; 22(5): e17361. Disponível em: https://doi.org/10.2196/17361

Boccolini, C.S., Boccolini, P.M., Monteiro, F.R., Venâncio, S.I. and Giugliani, E.R. (2017) Breastfeeding indicators trends in Brazil for three decades. Rev Saude Publica; 51:1-9. Disponível em: https://doi.org/10.11606/S1518-8787.2017051000029 
Dermatini, F. (2018) “Telegram chega a 200 milhões de usuários mensais”, Disponível em: $\quad$ https://canaltech.com.br/apps/telegram-chega-a-200-milhoes-de-usuariosmensais-110532

IBM (2019) “IBM Watson”, Disponível em: https://www.ibm.com/Watson/APIs

Nichols, T.R., Wisner, P.M., Cripe, G., et al. (2010) Putting the Kappa Statistic to Use, Qual Assur J 13, p. 57-61. Disponível em: https://doi.org/10.1002/qaj.48

O'Neill., T.A. (2017) An Overview of Interrater Agreement on Likert Scales for Researchers and Practitioners. Frontiers In Psychology, 8(1), p. 1-15. Disponível em: http://dx.doi.org/10.3389/fpsyg.2017.00777

Pressman, R. (2016), Engenharia de Software: Uma Abordagem Profissional, McGrawHill, Bookman, $8^{\mathrm{a}}$ edição.

Polit, D.F., and Beck, C.T. (2006) The content validity index: Are you sure you know what's being reported? critique and recommendations, Res Nurs Health; 29(5), p. 48997. Disponível em: https://doi.org/10.1002/nur.20147

Santos, I.S., Barros, F.C., Horta, B.L., Menezes, A.M.B., Bassani, D., Tovo-Rodrigues, L., et al. (2019) Breastfeeding exclusivity and duration: trends and inequalities in four population-based birth cohorts in Pelotas, Brazil, 1982-2015. Int J Epidemiol. 48(Suppl 1), p. 72-9.

Schincaglia, R.M., Oliveira, A.C., Sousa, L.M. and Martins, K.A. (2015) Práticas alimentares e fatores associados à introdução precoce da alimentação complementar entre crianças menores de seis meses na região noroeste de Goiânia. Epidemiol. Serv. Saúde, Brasília, 24 (3), p. 465-74.

Senna, A.F., Giugliani, C., Lago, J.C., Bizon, A.M., Martins, A.C., Oliveira, C.A., et al. (2020) Validation of a tool to evaluate women's satisfaction with breastfeeding for the Brazilian population. J Pediatr (Rio J); 96, p. 84-91

Silva, A.N., Santos, A.M.G., Cortez, E.A. and Cordeiro, B.C. (2015) Limites e possibilidades do ensino à distância $(\mathrm{EaD})$ na educação permanente em saúde: revisão integrativa. Ciência \& Saúde Coletiva, 20(4), p. 1099-107.

Silva, C.P.V., Fettermann, F.A., Assumpção, P.K., Rosa, A.B., Fernandes, M.N.S. and Donaduzzi, D.S.S. (2020) Aleitamento materno exclusivo na primeira hora de vida do recém-nascido. Saúde (Santa Maria), 46(1).

Tang, K., Gerling, K., Chen, W. and Geurts L. (2019) Information and Communication Systems to Tackle Barriers to Breastfeeding: Systematic Search and Review. J Med Internet Res; 21(9):e13947. Disponível em: https://doi.org/10.2196/13947

Vilar, T.M., Oliveira, I.K.F., Monteiro, N.V.N., Araújo, F.Y.G. and Carvalho, C.M.R.G. (2020) Educação em saúde e direito: em busca da proteção do aleitamento materno e dos direitos das gestantes em uma maternidade pública. Research, Society and Development; 9 (1), p. 6.

World Health Organization - WHO (2009) Infant and young child feeding. Geneva: World Health Organization. Disponível em: https://apps.who.int/iris/bitstream/handle/10665/44117/9789241597494_eng.pdf 